\title{
4. SEA ICE PHYSICS
}

\author{
Stephen Wells, ${ }^{2}$ Mark Tadross, ${ }^{2}$ and Shipboard Scientific Party ${ }^{3}$
}

\section{INTRODUCTION}

When plans for ODP Leg 151 were made, it was soon clear that the leg also would provide an excellent opportunity to collect data on ice properties, distribution, and movement for extended intervals. The role of sea ice and its snow layer in climate models is significant, its high surface albedo and its low thermal conductivity regulating the transfer of energy between the relatively warm ocean and the colder atmosphere. Its surface roughness aids momentum transfer between the two, and the processes leading to the formation of new ice are thought to be significant in the renewal of the majority of the planet's deep water. The extent of the seasonal ice cover is well documented, but the distribution of ice type, thickness, and features such as pressure ridging that modulate the above processes are not. The availability of an observational platform for an extended period due to Arctic sea ice edge in the Fram Strait and Yermak Plateau area during late summer 1993, therefore, created an opportune time to collect ice data.

\section{PURPOSE OF ICE SCIENTISTS SAILING ON FENNICA}

Satellite remote sensing has been proven as a useful tool for the extraction of geophysical parameters, offering systematic and repetitive coverage of areas of interest at a relatively low cost. It is well suited to the study of the polar regions where the monitoring of conditions on micro- and macroscopic scales is both difficult and expensive due to the harsh physical conditions encountered. In these regions cloud-penetrating microwave imagery is especially useful.

However, to successfully interpret these data, in-situ measurements of relevant physical properties are needed. These measurements are used to interpret simultaneously acquired satellite imagery. The objective is to extrapolate the results, thus greatly reducing the need for groundwork and greatly increasing the area that can be characterized. ODP Leg 151 had contracted ice forecasting based on satellite imagery; the participation of glaciologists on the accompanying ice-breaker Fennica offered a welcome opportunity to "groundtruth" some of the data.

\section{OBJECTIVES}

At the start of Leg 151 the objective of the ice program was to characterize ice properties observed in the Marginal Ice Zone (MIZ) and relate changes in these properties to the radar backscatter signature observed by the ERS-1 satellite. This objective required deployment onto floes greater in size than $200 \mathrm{~m}$, because anything smaller

'Myhre, A.M., Thiede, J., Firth, J.V., et al., 1995. Proc. ODP, Init. Repts., 151: College Station, TX (Ocean Drilling Program).

${ }^{2}$ Scott Polar Research Institute, University of Cambridge, Lensfield Road, Cambridge CB2 IER, United Kingdom.

'Shipboard Scientific Party is as given in the list of participants preceding the Table of Contents. than this is unlikely to appear with any clarity in the Synthetic Aperture Radar (SAR) imagery, hence restricting identification of the sampling scene.

As the floe size encountered was considerably less than $200 \mathrm{~m}$, we decided to concentrate on work that could be used to interpret passive microwave imagery. Our intention was to quantify the properties of areas of open water and surface meltwater that would affect passive microwave algorithms for sea ice concentration.

\section{REMOTE SENSING}

Satellite imagery was available during Leg 151 from the satellites and sensors listed in Table 1 (Massom, 1991). Low- and high-resolution ERS-1 SAR imagery of the operational area was obtained from the ground station at Troms $\emptyset$, Norway. The SAR imagery received aboard Fennica was low resolution with additional interpretation overlaid (Fig. 1). The SSM/I imagery received aboard the ship was in the form of ice concentration maps (Fig. 2) having already had a concentration algorithm applied.

Also received aboard the ship were visible and infrared band images from the U.S. National Oceanic and Atmospheric Administration (NOAA) satellites 10 and 11 (Advanced Very High Resolution Radiometer [AVHRR] sensor), with ground resolution of $4 \mathrm{~km}$ and swath width of $4000 \mathrm{~km}$, and the Russian Meteor Series 2 and 3 satellites (Fig. 3). Unlike the two microwave sensors, SAR and SSM/I, which are unaffected by cloud cover and thus well suited for coverage of the polar regions, the NOAA AVHRR and Meteor sensors work in the visible and infrared bands and are reliant on clear skies to give useful ground coverage.

Both the SAR and the SSM/I imagery were available to the ship by computer download via a modem and satellite link to the Nansen Environmental Remote Sensing Center (NERSC) in Bergen, Norway. The images were transferred from the UNIX system at NERSC in the form of compressed image (JPEG format) files and printed on

Table 1. Satellite imagery used during Leg 151.

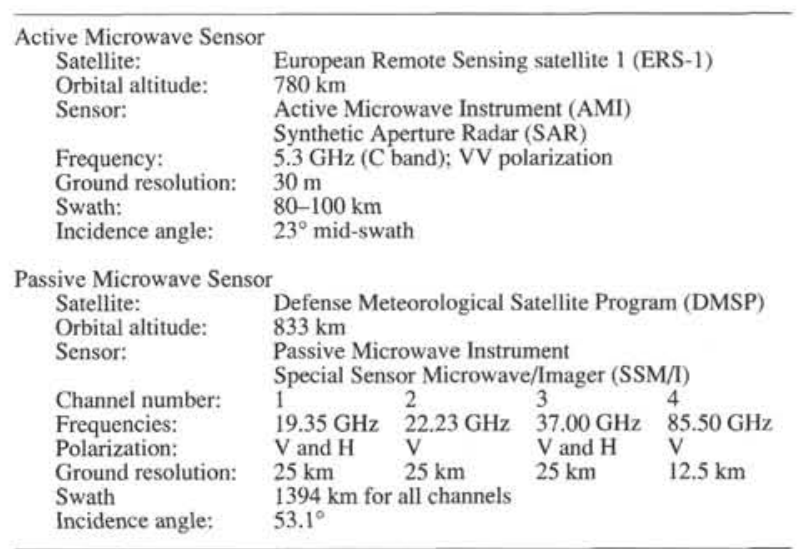




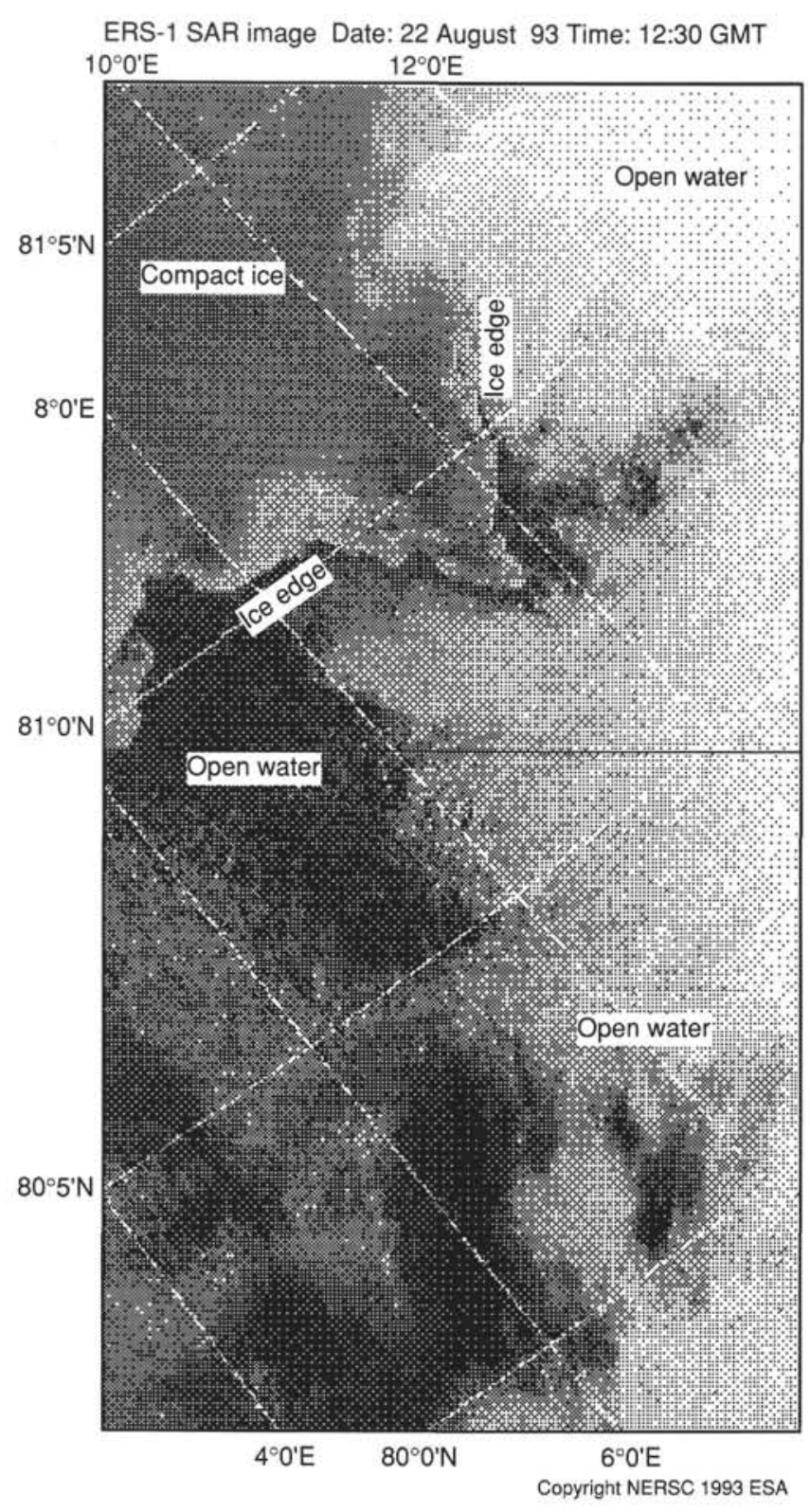

Figure 1. SAR image, date and time as shown, received aboard Fennica. Interpretation was added by NERSC before transmission to the ship. When the image was taken, the ship was at $81^{\circ} 02.8^{\prime} \mathrm{N}, 07^{\circ} 21.8^{\prime} \mathrm{E}$. Courtesy of NERSC.

the ship's laser printer. In high latitudes, when links to the INMARSAT system were less reliable, copies of the imagery were sent by NERSC via telefax.

The NOAA AVHRR and Meteor imagery was received aboard Fennica as Automatic Picture Transmission (APT) imagery directly from the satellite using WEATHERTRAC II software.

\section{THEORY}

Passive microwave ice concentration algorithms previously have relied on the form first developed by Cavalieri et al. (1984), known as the NASA Team algorithm. The vertical and horizontally polarized $19 \mathrm{GHz}$ and $37 \mathrm{GHz}$ channels are used to calculate a polarization

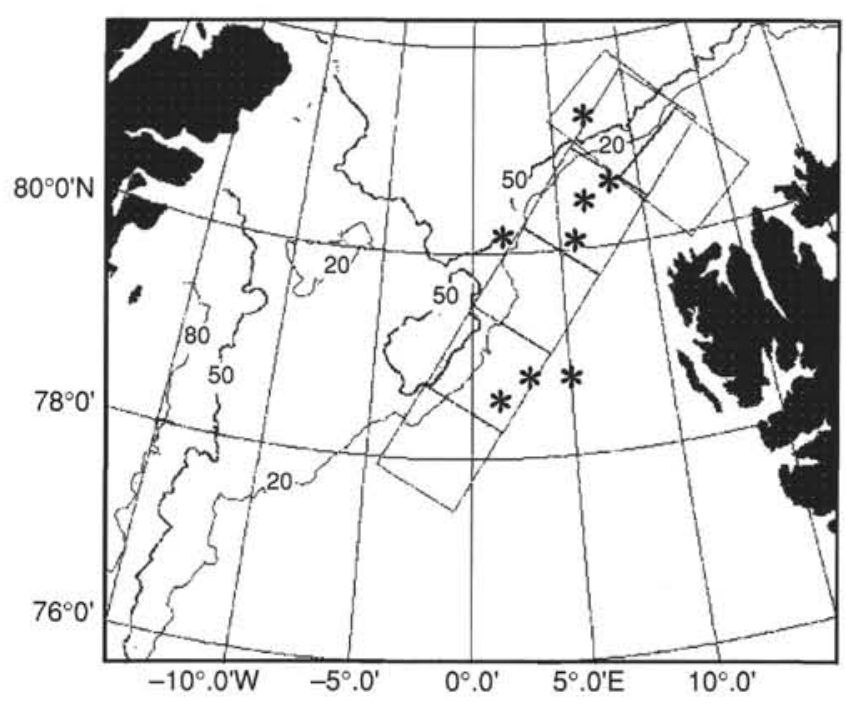

Figure 2. Total ice concentration in \% for SSM/I image of 23 August 1993 , with SAR outlines for 22 August 1993. Courtesy of NERSC. Asterisks indicate proposed drill sites.

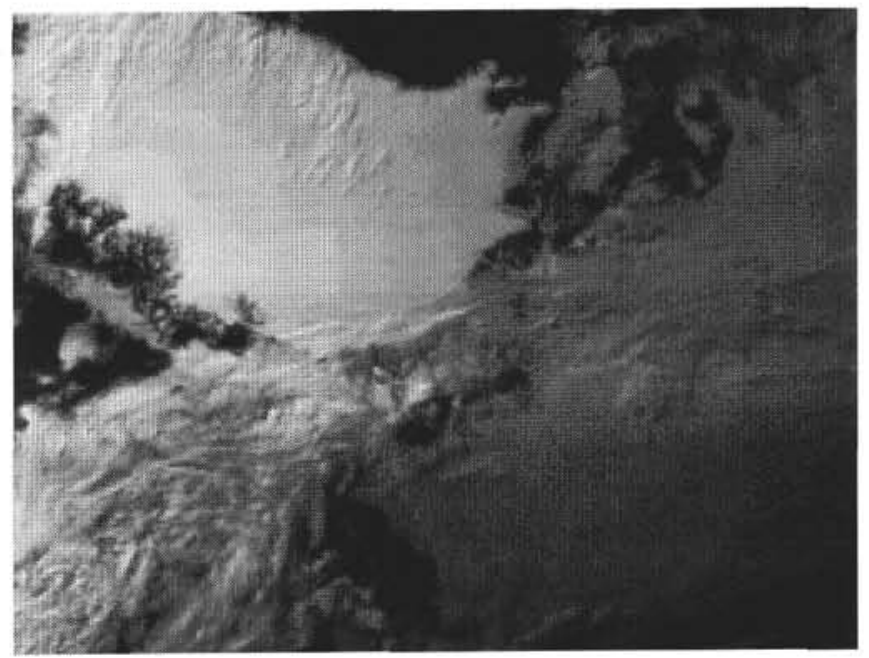

Figure 3. Russian Meteor satellite image received by APT at 1500 GMT on 31/08/93. Greenland, the North coast of Svalbard, and limit of the Arctic pack ice can be seen.

ratio (PR) and spectral gradient ratio (GR) (calculation details given in Cavalieri et al., 1984). Variations in PR generally reflect changes in ice concentration (and distinguish ice from open water), whereas the GR is used to distinguish between first-year and multi-year ice. The need for a mixing algorithm arises because of the large areal coverage of each pixel in an SSM/I image $(25 \mathrm{~km}$ by $25 \mathrm{~km}$ ). This results in open water and both ice types contributing to the measured brightness temperature.

Flooded ice also can be treated as a simple model involving a brine (depending on the salinity of meltwater) layer on the ice surface. The emissivity changes at different frequencies and the susceptibility to changes in brine depth are also frequency dependent. Using this two-layer model, the brightness temperature of flooded ice can be calculated. NOAA AVHRR imagery and the phenomenon of sun glint (Goroch and Fett, 1993) may be used to quantify the amount of flooded ice. 


\section{Electrical Properties of Sea Ice}

Physical properties affecting the microwave signature of sea ice and its snow cover are roughness, wetness, grain size, density, and salinity. The frequency, polarization and incidence angle of the sensing radiation also determine the mechanism by which the two interact.

Passive microwave instruments quantify the intensity of emitted radiation by converting it into the brightness temperature $\left(T_{b}\right)$ of an object. This quantity is related to the physical temperature by the equation:

$$
T_{b}=\varepsilon \cdot T_{s}
$$

where $\varepsilon$ is the emission coefficient of the object and $T_{s}$ is the physical surface temperature. $\varepsilon$ is frequency and incident angle (?) dependent and quantifies the similarity to a black body emitter $(\varepsilon=1)$.

Active microwave instruments transmit pulses that are reflected and scattered by a target. The signal received by the sensor is a measure of the amount of backscattered radiation and is quantified by the backscatter coefficient $\left(\varepsilon_{0}\right)$, which is defined in terms of the incident and scattered electric fields.

The relative permittivity is a complex number that characterizes the electrical properties of a medium:

$$
e=e^{\prime}+j e^{\prime \prime},
$$

where $j$ is the square root of $-1, e^{\prime}$ is the dielectric constant and $e^{\prime \prime}$ the dielectric loss factor. The dielectric constant gives the contrast with respect to free space $\left(0<e^{\prime}<1\right)$, whereas the loss factor gives the electromagnetic loss of the material (conversion to other forms of energy).

The penetration depth $\left(\varepsilon_{p}\right)$ is the distance a wave travelling into a medium reaches before the ratio of its power to its initial power falls to the value $1 / e(e=2.718)$.

$$
P\left(\varepsilon_{p}\right) / P(0)=1 / e .
$$

$P(0)$ is the power transmitted at the medium boundary (in the case of sea ice remote sensing, the air/snow or air/ice boundary) (Carsey, 1992; Ulaby et al., 1982).

\section{Variations in Microwave Signatures Because of Seasonal Change}

The microwave properties of sea ice vary each year because of changing climatic conditions. In winter the low temperatures mean that the snow and upper layers of sea ice remain well below $0^{\circ} \mathrm{C}$, keeping them free of liquid water. Under these conditions, first-year ice and multi-year ice (ice that has survived more than one summer's melt) appear distinct from one another in both passive and active microwave imagery. Due to brine, trapped in pockets during the formation process, first-year ice has a high dielectric loss factor, which acts to decrease the penetration depth, thus lowering the radar backscattering coefficient. Conversely, multi-year ice is almost salt free with a low loss factor and therefore a higher penetration depth. Inhomogeneities in the ice, such as air bubbles caused by brine drainage, increase the backscatter. In the passive microwave case, increased backscatter leads to a lower emissivity value for multi-year ice (Kim et al., 1984).

The situation during the summer melt season makes it much more difficult to distinguish between first-year and older ice. The presence of volumetric water in the snow cover and meltwater on the ice surface leads to a reduction in the penetration depth of microwaves. Radar backscatter becomes dominated by surface scattering, regardless of the physical structure of the underlying ice. The emissivity value of both first-year and multi-year ice becomes dependent on the overlying snow cover or top few centimeters of waterlogged ice. This value is close to unity and results in concentration algorithms (which rely on the difference in emissivity between ice and water) underestimating the total ice concentration. A detailed discussion of seasonal variability in sea ice signatures is given by Onstott et al. (1987).

\section{DATA COLLECTION}

\section{Ice Surface}

Ice surface parameters were measured where appropriate (Table 2). The "snow fork" is an instrument developed to measure snow density and wetness profiles by means of an electric field generated be-

\begin{tabular}{|c|c|c|}
\hline Variable & Units & $\begin{array}{c}\text { Instrument/ } \\
\text { data collection }\end{array}$ \\
\hline Snow temp. profiles & ${ }^{\circ} \mathrm{C}$ & Temperature probe \\
\hline Snow depth & $\mathrm{cm}$ & Meter stick \\
\hline Snow density & $\mathrm{g} / \mathrm{cm}^{3}$ & Snow fork \\
\hline Snow wetness & Volumetric \% & Snow fork \\
\hline Snow grain size & $\mathrm{mm}$ & Observational \\
\hline Melt pool size, depth & $\mathrm{m}$ & Tape measure \\
\hline
\end{tabular}
tween two metal prongs.

Table 2. Ice surface parameters measured during Leg 151.

\section{Ice Cores}

Cores were taken using a 1-m-long, 3.25-in. diameter Cold Regions Research Laboratory (CRREL) type corer barrel driven by a post hole drill engine. Core temperatures were obtained by drilling into the core at $10-\mathrm{cm}$ intervals, using a hand drill, and inserting the temperature probes into the resulting holes. Where necessary (often the core split into suitable lengths), the core was cut into $10-\mathrm{cm}$ lengths using a hand saw. An estimate of the mass of ice in these sections was obtained using a simple balance. The lengths were allowed to melt and the conductivities of the resulting solutions measured.

\section{Ice Observations Log}

A record of ice conditions observed during Leg 151 was made in accordance with the protocols laid out for the Greenland Sea Project (Garrity and Ramseier, 1992). While moving in continuous cover, we made observations every hour, consisting of written notes and photographs (mostly black-and-white) of the ice conditions together with a record of the ship's position and the prevailing atmospheric conditions. At other times, observations were made when/where conditions changed. Where possible, the following parameters were noted: total cover; floe size and age distribution; snow and ice depth; presence of surface features (melt pools, thaw holes, ridging, "dirty" ice, etc.). These observations were supplemented with notes, copies of the ship's ice charts, sketches and photographs of the ship's radar screens, etc., when conditions did not allow visual observations.

\section{RESULTS}

\section{Summary}

Due to the nature of the deployment of Fennica, the sampling program was necessarily disrupted. In general, where time/permission had been granted for science from Fennica, if the ship was in an area at the time of an ERS-1 SAR pass, physical measurements were per- 
formed on the ice (if any) present at that location. Physical sampling was conducted at six sites.

On two occasions when time/permission had been granted for science from Fennica and there was not a coincident SAR pass, an attempt was made to characterize the ice situation over as large an area as possible by making continuous, detailed ice observations while the ship moved on a transect through an area. An exception to this arrangement was made if the ice type encountered in an area had not been physically sampled previously.

\section{Ice Observations}

Written ice observations have been combined with coincident imagery, ship's ice charts, and 33 black-and-white photographs to form an ice observations log for Leg 151 (see "Sea Ice Observations Log" chapter, this volume). This will form a useful comparative reference when the SAR and SSM/I imagery is examined post-cruise.

Because of the many days of poor visibility, much reliance was placed on the ship's X and S band radars for determining the ice edge position. The ship's officers estimate that with a compact edge in calm sea conditions, the edge could be charted correctly to within a cable (one-tenth of a nautical mile). In rough seas, with a diffuse ice edge (the worst case), this accuracy could fall to $0.5 \mathrm{nmi}$ or worse.

One distinct floe was encountered repeatedly on the Fennica's ice watches in the region North of $81^{\circ} \mathrm{N}$. Its size and freeboard provided a strong radar return at distances of up to $10 \mathrm{nmi}$, even when it was located inside the ice edge. This may have been a piece of sikussakfast ice of great thickness- broken off from the northern coasts of Greenland or the Canadian Arctic. Its appearance closely matches the description given by Wadhams (1986b) of sikussak-type ice seen in Greenland fjords, at $82^{\circ} 24^{\prime} \mathrm{N}, 16^{\circ} 22^{\prime} \mathrm{W}$, in 1980 . Unfortunately, prevailing conditions never permitted any surface sampling.

\section{Ice Cores and Sampling}

Thirty-four 1-m-deep ice cores were taken from floes at six locations. Salinity results obtained match those expected from first-year (typically $3 \mathrm{ppt}$ ) and multi-year (typically 0-2 ppt) Arctic ice. On multi-year floes, cores taken from pressure ridges showed almost zero salinity; those taken from hummocks displayed values typical of cores from areas of little surface relief. "Dirty" ice samples were collected on two occasions for analysis of their sediments.

\section{SURFACE MEASUREMENTS}

\section{Melt Pools}

Water samples from 69 melt pools were taken for salinity measurements. Results show a range from 0.1 to $12.5 \mathrm{ppt}$. The highest values came from pools noted as being close to the edge of floes and/ or connected by drainage channels to the sea. Measured depths were in the range 0.05 to $0.30 \mathrm{~m}$. Many pools encountered later in the cruise were frozen.

\section{Wetness and Density}

Typical "snow fork" values for permittivity and wetness are given in Table 3. Obtained from a snow profile sampled on the surface of a multi-year floe, they are indicative of a new and dense, dry snow cover.

\section{POST-CRUISE PROCESSING}

The variability of ice concentration estimates from passive microwave data is thought to be caused in part by changing pure type sig-
Table 3. Snow density and wetness profile, taken on 9 Sept. 1993.

\begin{tabular}{cccccc}
\hline \multirow{2}{*}{$\begin{array}{c}\text { Depth } \\
(\mathrm{cm})\end{array}$} & \multicolumn{2}{c}{ Permittivity } & $\begin{array}{c}\text { Wetness } \\
\text { (vol\%) }\end{array}$ & $\begin{array}{c}\text { Density } \\
\left(\mathrm{g} / \mathrm{cm}^{3}\right)\end{array}$ & $\begin{array}{c}\text { Wetness } \\
(\mathrm{wt} \%)\end{array}$ \\
\hline 0 & 1.25 & 0.006 & 0.69 & 0.104 & 06.5 \\
2 & 1.27 & 0.000 & 0.00 & 0.147 & 00.0 \\
4 & 1.36 & 0.002 & 0.24 & 0.187 & 01.2 \\
6 & 1.35 & 0.003 & 0.36 & 0.179 & 01.9 \\
8 & 1.34 & 0.001 & 0.12 & 0.183 & 00.6 \\
10 & 1.36 & 0.002 & 0.24 & 0.190 & 01.2 \\
12 & 1.39 & 0.000 & 0.00 & 0.211 & 00.0 \\
14 & 1.37 & 0.001 & 0.12 & 0.197 & 00.5 \\
16 & 1.32 & 0.004 & 0.48 & 0.157 & 03.0 \\
18 & 1.35 & 0.004 & 0.48 & 0.171 & 02.7 \\
20 & 1.39 & 0.000 & 0.00 & 0.211 & 00.0 \\
22 & 1.52 & 0.000 & 0.00 & 0.270 & 00.0 \\
24 & 1.47 & 0.003 & 0.38 & 0.234 & 01.6 \\
\hline
\end{tabular}

natures: those of open water, first-year ice, and multi-year ice. Evidence for this comes from the observations during summer melt of a decrease in ice concentration estimates and a corresponding increase in brightness temperature. First-year and multi-year ice both have higher emissivities than open water, indicating that the two should vary in phase, the result of using fixed global signatures. The observations made from Fennica of physical variables and the spatial distribution of the floes will be tied to the changes in recorded brightness temperature. Any predominant effects by one or more variable, we hope, will be distinguishable.

A model can be used to predict $T_{b}$ signatures (Klein and Swift, 1977 ) and compared with those observed. The main problem with this is the need to quantify the ice distribution over an SSM/I pixel. This can be done using visible AVHRR or Landsat imagery, but is not likely to be of use given the cloud coverage during the cruise. Investigation of the high-resolution SAR imagery may provide useful concentration data that can be applied over the $25-\mathrm{km}$ SSM/I pixels. However, the broken nature of the MIZ can produce a backscatter scenario so complicated that the concentration data are useless. The SAR data will give an accurate position of a compact ice edge for comparison both with that given by the SSM/I and that observed from the ship. Initial impressions suggested that the $50 \%$ ice concentration given by the SSM/I was a better indicator of the ice edge than were other concentrations. Actual concentrations in the range 20\%-30\% tended to show large variability in observed signature.

The variability of the multi-year signature is thought to be because of deviations from the ideal situation (e.g., appearing as firstyear ice because of seawater intrusion into the snow-ice interface) (Comiso, 1990). SAR offers the possibility of independent, multiyear concentration estimates for comparison (away from the broken $\mathrm{MIZ}$ ), and the observed variables may point to contributory effects.

\section{CONCLUSIONS}

No conclusions can be drawn with regard to measurements performed during the cruise until the full resolution imagery is examined in detail.

However, some initial comment may be made on the usefulness of SAR and SSM/I data for planning and navigation during operations such as Leg 151. During the planning stages of the operation, the ability to look at the annual and monthly variations in sea-ice cover, much of which is satellite-generated information, may be of great value in assessing the operation's feasibility.

During the operation, however, with the availability of an ice-capable ship such as the Fennica and the relative proximity of all the drill locations, the SAR and SSM/I were less useful than anticipated. The imagery was helpful in approximating the location of the ice edge at the start of operations in the area, but the ability of Fennica to give detailed, continuous ice reports over the sites in question with- 
in a few hours of a request, together with its ability to monitor local conditions such as drift, made the imagery almost redundant.

In the conditions often encountered, for example with a windscattered MIZ made up of small floes bounded by areas of windroughened open water, the SAR imagery was of limited value. The sometimes rapid changes in ice extent associated with these condi- tions meant that even "near-real-time" imagery, often delayed by communications problems associated with using INMARSAT at high latitudes, was out of date.

\section{Ms 151IR-104}

